\title{
Correction to: Caregiver burden and its related factors in advanced Parkinson's disease: data from the PREDICT study
}

\author{
Alessandro Tessitore ${ }^{1} \cdot$ Pietro Marano $^{2} \cdot$ Nicola Modugno $^{3} \cdot$ Francesco E. Pontieri $^{4} \cdot$ Nicola Tambasco $^{5}$. \\ Margherita Canesi ${ }^{6} \cdot$ Anna Latorre $^{7} \cdot$ Leonardo Lopiano $^{8} \cdot$ Mariachiara Sensi $^{9} \cdot$ Rocco Quatrale $^{10}$. \\ Paolo Solla ${ }^{11}$. Giovanni Defazio ${ }^{11}$ - Gabriella Melzi ${ }^{12}$ - Anna Maria Costanzo ${ }^{12}$. Giuliana Gualberti ${ }^{12}$. \\ Umberto di Luzio Paparatti ${ }^{12}$. Angelo Antonini ${ }^{13}$
}

Published online: 20 May 2020

(C) The Author(s) 2020

\section{Correction to: Journal of Neurology (2018) 265:1124-1137 https://doi.org/10.1007/s00415-018-8816-9}

The original version of this article unfortunately contained a mistake. In Fig. 2, there are some mistakes that author would like to correct. In particular, in the part A of the figure we have added in the explanation of the figures that in the SOC group there were 8 subjects with missing data. In the part 2B we have included the statistical differences between groups while in the part $2 \mathrm{C}$ in the LCIG group, the subjects reporting no change in the CGI assessment was zero.

The corrected Fig. 2 with caption is placed.
The original article can be found online at https://doi.org/10.1007/ s00415-018-8816-9.

Gabriella Melzi

gabriella.melzi@abbvie.com

1 First Division of Neurology, University of Campania, "Luigi Vanvitelli", Napoli, Italy

2 Nuova Casa di Cura D’Anna, Palermo, Italy

3 Neurology Unit, IRCCS Neuromed, Pozzilli, IS, Italy

4 Department NESMOS, "Sapienza” University, Sant'Andrea Hospital, Rome, Italy

5 Perugia General Hospital and University of Perugia, Perugia, Italy

6 Centro Specialistico Ortopedico Traumatologico G. Pini-CTO Milano, Milan, Italy
Sapienza University, Rome, Italy

8 Department of Neuroscience, University of Torino, Azienda Ospedaliero-Universitaria Città della Salute e della Scienza di Torino, Turin, Italy

9 Neurology Unit, Hospital Sant'Anna Ferrara, Ferrara, Italy

10 Neurology Unit, Hospital dell'Angelo, Mestre, VE, Italy

11 Neurology Unit, Policlinico Universitario Monserrato, Cagliari, Italy

12 AbbVie Srl, SR 148 Pontina, 04011 Campoverde, LT, Italy

13 Parkinson and Movement Disorders Unit, Department of Neuroscience, University of Padua, Padua, Italy 
(A)

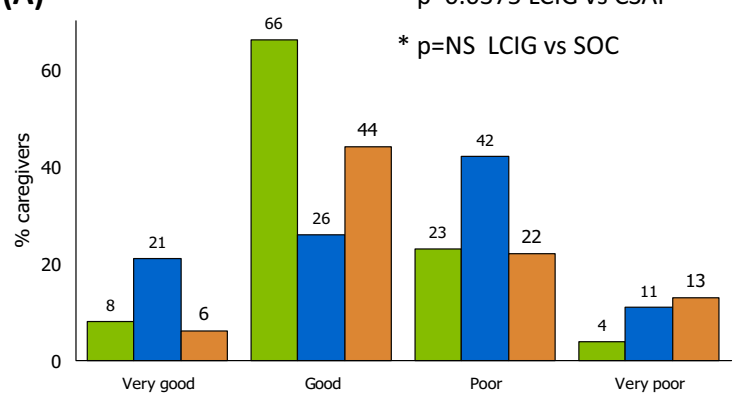

(C)

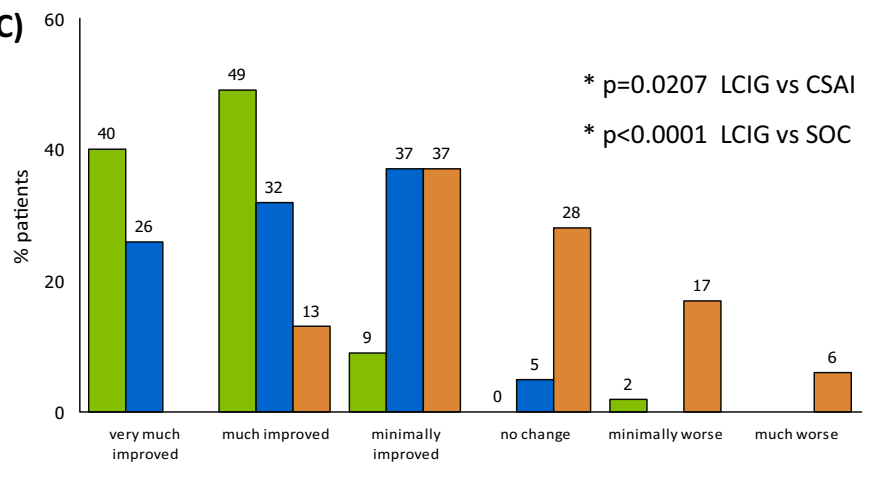

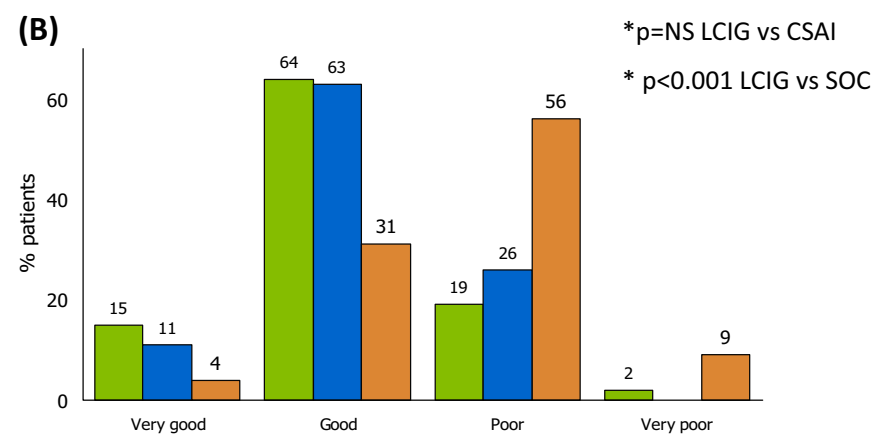

$\square$ DUO $\square$ APO $\square$ SOC
Fig. 2 Global judgment of caregivers (a) and patients (b) on their current quality of life compared to previous standard treatment and Clinical Global Impression-Global Improvement Scale (CGI-I) by physician (c); * Chi-square test (a, b) or Fisher exact test (c); in $\mathbf{a}, \mathbf{b}$

Open Access This article is licensed under a Creative Commons Attribution 4.0 International License, which permits use, sharing, adaptation, distribution and reproduction in any medium or format, as long as you give appropriate credit to the original author(s) and the source, provide a link to the Creative Commons licence, and indicate if changes were made. The images or other third party material in this article are included in the article's Creative Commons licence, unless indicated otherwise in a credit line to the material. If material is not included in data were grouped as "very good + good" vs "poor + very poor"; in c data were grouped as "improved" vs "no change or worse"; 8 missing data for SOC caregivers in a

the article's Creative Commons licence and your intended use is not permitted by statutory regulation or exceeds the permitted use, you will need to obtain permission directly from the copyright holder. To view a copy of this licence, visit http://creativecommons.org/licenses/by/4.0/. 